\title{
Physical limits for high ion charge states in pulsed
}

\author{
discharges in vacuum
}

\author{
Georgy Yu. Yushkov \\ High Current Electronics Institute, Russian Academy of Sciences, Tomsk, 634055, \\ Russia
}

André Anders

Lawrence Berkeley National Laboratory, Accelerator and Fusion Research Division, Berkeley, California 94720, USA

Submitted to the Journal of Applied Physics, revised Dec. 2008, accepted Jan. 2009

\section{ACKNOWLEDGMENT}

This work was supported by the U.S. Department of Energy, Initiatives for Proliferation Prevention, under Contract No. DE-AC02-05CH11231 with the Lawrence Berkeley National Laboratory.

\section{DISCLAIMER}

This document was prepared as an account of work sponsored by the United States Government. While this document is believed to contain correct information, neither the United States Government nor any agency thereof, nor The Regents of the University of California, nor any of their employees, makes any warranty, express or implied, or assumes any legal responsibility for the accuracy, completeness, or usefulness of any information, apparatus, product, or process disclosed, or represents that its use would not infringe privately owned rights. Reference herein to any specific commercial product, process, or service by its trade name, trademark, manufacturer, or otherwise, does not necessarily constitute or imply its endorsement, recommendation, or favoring by the United States Government or any agency thereof, or The Regents of the University of California. The views and opinions of authors expressed herein do not necessarily state or reflect those of the United States Government or any agency thereof or The Regents of the University of California. 


\title{
Physical limits for high ion charge states in pulsed discharges in vacuum
}

\author{
Georgy Yu. Yushkov \\ High Current Electronics Institute, Russian Academy of Sciences, Tomsk, 634055, \\ Russia
}

\section{André Anders}

Lawrence Berkeley National Laboratory, Berkeley, California 94720, USA

\begin{abstract}
Short-pulse, high-current discharges in vacuum were investigated with the goal to maximize the ion charge state number. In a direct extension of previous work [Appl. Phys. Lett. 92, 041502 (2008)], the role of pulse length, rate of current rise, and current amplitude was studied. For all experimental conditions, the usable (extractable) mean ion charge state could not be pushed beyond 7+. Instead, a maximum of the mean ion charge state (about $6+$ to $7+$ for most cathode materials) was found for a power of 2-3 MW dissipated in the discharge gap. The maximum is the result of two opposing processes that occur when the power is increased: (i) the formation of higher ion charge states, and (ii) a greater production of neutrals (both metal and non-metal), which reduces the charge state via charge exchange collisions.
\end{abstract}




\section{INTRODUCTION}

It is well known that copious amounts of multiply charged ions are produced in cathodic vacuum arcs. ${ }^{1-5}$ The charge states can be shifted higher by a number of measures like the application of a magnetic field. ${ }^{6,7}$ This was shown to be related to greater power dissipation because the burning voltage of the discharge, measured between anode and cathode, is greatly enhanced. Furthermore, it is also known that charge states can be shifted to lower values by the interaction with background gas neutrals ${ }^{8,9}$ and neutrals (vapor) of the cathode material. ${ }^{10}$

In a previous short publication of preliminary results, ${ }^{11}$ we reported on an attempt to utilize this knowledge and maximize the ion charge states. Emphasis was put not only on ion production but on ion extraction from such plasma because transient production without extraction is not interesting to practical application like ion implantation or surface modification and film deposition. The term "extraction" is here broadly used to include the use of ions via gridded extractor or via the sheath of a substrate.

We have shown ${ }^{11}$ that short pulse, high-current operation allowed us to obtain mean charge states between 6 and 7, with the highest charge state in the distribution being 10, or even 11 in the case of gold. The discharges are characterized by a timedependent high burning voltage, typical for sparks, and therefore the type of discharge is best characterized as a transient between vacuum sparks (high burning voltage) and vacuum arcs (low burning voltage of $\sim 20 \mathrm{~V}$ ).

In this more comprehensive contribution, we follow up on those lines of thought and explore how much further we could push the production of elevated charge states. The logical approach is to try even shorter pulses with higher currents and steeper pulse shapes. We will explore the role of the rate of current rise, if any, and why there appears to be a current optimum. We will show that we encounter some limits and that those limits appear to be rather fundamental. As a result, we will show that there is a range of optimum discharge conditions that delivers a maximum of the average charge state number. 


\section{EXPERIMENTAL}

As with the preliminary report, ${ }^{11}$ the experiments were carried out at the modified vacuum arc ion source "Mevva V" at Berkeley Lab (the original name is derived from "metal vapor vacuum arc"). ${ }^{3}$ The cathode was a $6.25 \mathrm{~mm}$ rod coaxially placed in an annular copper tube of $25 \mathrm{~mm}$ inner diameter, which serves as the anode (Fig. 1). The pulse forming network supply, usually employed for the ion source, was replaced by circuits designed for short pulses of much higher current. In this report, two new circuits were built using a high voltage, low inductance capacitor of either $0.15 \mu \mathrm{F}$ or $0.45 \mu \mathrm{F}$. For comparison, a circuit with a capacitor of $11.6 \mu \mathrm{F}$ was used for the preliminary report. ${ }^{11}$ In all cases, the selected capacitor was charged by a direct current (DC) high voltage power supply. On the discharge side, short, thick (low-inductance) cables were used to make the connections to anode and cathode. The discharge was triggered by the standard Mevva-V trigger pulse, which is a high voltage pulse of $12 \mathrm{kV}, 20 \mathrm{~A}$, delivered via a high-voltage step-up transformer. The nominal voltage is here the open-circuit voltage, which breaks down once plasma is formed. The power dissipated by the trigger discharge is much smaller than the power of the main discharge.

The peak discharge current could be selected simply by adjusting the capacitor's charging voltage, which in these experiments could be as high as $30 \mathrm{kV}$, i.e. much higher than before. However, as we will show, a charging voltage as high $15 \mathrm{kV}$ is more than sufficient to demonstrate the limitations of the approach, and therefore all results presented here are for a charging voltage of $15 \mathrm{kV}$ or less. Pulse current and burning voltage were recorded by a fast digital oscilloscope (Tektronix TDS 5054B). The power was readily determined by multiplication of the time-dependent voltage and current channels - this can be done using the mathematics function of the oscilloscope, or via software after data export to a personal computer. Table 1 summarizes the maximum parameters of charging voltage, peak current, and peak power when using a gold cathode. The rate of current rise is also included; for simplicity we selected the time needed for the current to rise from $10 \%$ to $90 \%$ of the maximum value, which may be denoted as $(\Delta I / \Delta t)_{10 \rightarrow 90}$. Examples of current, voltage, and power are shown in Fig. 2 for the each of the three circuits. 
The plasma is produced at cathode spots on the front face of the cathode rod. It expands through the anode opening into a larger cavity until it arrives, after $70 \mathrm{~mm}$ of flight, at the multi-aperture extractor grid of the three-grid, acceleration-deceleration extraction system. ${ }^{3}$ The extraction voltage was kept constant at $30 \mathrm{kV}$. The system was cryogenically pumped to a base pressure of about $1 \cdot 10^{-4} \mathrm{~Pa}$. After extraction, the ion beam propagated to the gate of the time-of-flight (TOF) spectrometer located $0.7 \mathrm{~m}$ downstream. The central beam stop of the gate arrangement can be used as an ion beam monitor (Fig. 3). The area of the beam stop is $20 \mathrm{~cm}^{2}$, and the recorded current is the sum of ion current and current of secondary electrons. Due to the spread in velocity of the plasma ions before extraction, the ion beam pulse appears much broader than the discharge pulse (Fig. 3). When a 150 ns gate pulse is applied to the gating plates, a portion of the broad beam is deflected toward a magnetically suppressed Faraday cup, the TOF detector, which is located $1.03 \mathrm{~m}$ from the TOF gate.

Several cathode materials have been used: Ag, Er, Cu, Au, Pt, Ta. They all showed similar behavior, and therefore it is justified to focus on representative examples. We pick gold $(\mathrm{Au})$ and platinum $(\mathrm{Pt})$ because for those elements we do not need to worry much about oxides on their surface, which helps with the identification of peaks in the TOF spectrum.

\section{RESULTS}

An example of a TOF spectrum is shown in Fig. 4. The new experiments show that, compared to the preliminary report, ${ }^{11}$ the charge states are not further enhanced even as we use shorter pulses and higher currents. The dependence on the discharge current is similar to what was reported for the slightly longer pulses (Figs. 5 and 6). Increasing the current beyond a certain optimum does not increase the charge state, rather they are reduced. Under optimum conditions, one can find charge states as high as $10+$. Charge states 4+ and 5+ can be considered "medium" in the context of this research: they show a non-monotonic dependence on the discharge current. At high currents (many kA), the fraction of low charge state metal ions increases and many non-metallic contaminations such as $\mathrm{H}^{+}, \mathrm{H}_{2}{ }^{+}, \mathrm{C}^{3+}, \mathrm{C}^{2+}, \mathrm{C}^{+}, \mathrm{O}_{2}{ }^{+}, \mathrm{O}^{+}$show up even for gold and platinum cathodes. 
Some researchers suggested that the rate of rise may be a valuable parameter characterizing the mean and maximum charge state that can obtained. Using the set of available data we plotted the mean charge state as a function of rate of current rise to find that the rate of rise is not suited to predict a mean charge state (Fig. 7).

A more physical approach is to consider the energy balance in the process of ion formation. We would expect that greater power density leads to higher charge states. We plot the mean charge state data as a function of peak power dissipated in the

discharge gap, $\hat{P}=\max [I(t) \times V(t)]$. Interestingly, there appears a maximum for all conditions at about $3 \mathrm{MW}$, as illustrated for the example of gold in Fig. 8.

\section{DISCUSSION}

The last observation, displayed in Fig. 8, is the main result of this contribution and it deserves more consideration. In the first part of the curves, increasing power dissipation leads to higher charge states, something one would intuitively expect given the energy demands in the production of higher charge states. The second, decreasing part of the curves (at high power) is less obvious. We should recall that the TOF spectrum at high voltage and current indicated a greater presence of lower metal charge states as well as contaminants (Fig. 4). The non-metallic contamination of the plasma can be caused by the interaction of an increasingly intense plasma flow from the cathode to the other components of the system, like anode, walls of the plasma expansion cavity, and the first grid of the ion extraction system. Once more non-metallic neutrals are in the volume, higher charge states are preferentially annihilated. ${ }^{10}$

Another clue brought the observation of the cathode itself. Prolonged operation by cathode spots produces a "well-arced" surface characterized by a metallic but slightly dull appearance due to roughness. In contrast, the surface of the cathodes used for the high current (high power) experiments showed signs of melting. This was true for all cathodes tested. If we pick the case of gold, for example, to be consistent with Fig. 8, we should consider evaporation from the cathode assuming the temperature reached melting temperature. The flux of evaporated atoms can be related to the material's equilibrium vapor pressure, $p_{\text {vapor }}$, by 


$$
J_{0}^{\text {evap }}=\frac{p_{\text {vapor }}\left(T_{c}\right)}{\sqrt{2 \pi m_{a} k T_{c}}} .
$$

It is known that the vapor pressure increases approximately exponentially with surface temperature and goes smoothly through the melting temperature; material-dependent data curves were tabulated by Honig. ${ }^{12}$ Table B1 in ref. ${ }^{5}$ lists the temperature for which the vapor pressure exceeds $1 \mathrm{~Pa}$; in the case of gold we find $1646 \mathrm{~K}$ which is not much higher than the melting temperature of $1337 \mathrm{~K}$. Therefore, as the cathode surface become very hot and even melts at high power, large amounts of metal vapor are produced into which the plasma expands containing high charge states. Charge exchange collisions of the type

$$
M e^{Q+}+M e \rightarrow M e^{(Q-1)+}+M e^{+}
$$

preferably reduce the highest charge states and increase the population (density) of singly charged metal ions.

The temperature of the cathode surface (outside the active cathode spots) is largely determined by ion bombardment heating which depends on the ion current density (which in turn depends on the plasma density), sheath voltage and ion charge state. As greater discharge currents are used via higher charging voltage, more cathode spots are active, producing more plasma and therefore the large-area heating of the cathode surface increases. The heat conduction equation ${ }^{13}$ and details of the heating and cooling processes are involved and discussed elsewhere. ${ }^{5}$ Here it is sufficient to emphasize that transient, large area melting of the surface necessarily implies the production of high density of metal neutrals. Therefore, increasing the power also necessarily increases the density of neutrals right near the cathode surface, which prevents further enhancement of the mean charge state of extractable or useable ions. A fresh-deposited metal surface acts as a getter for residual gas which can be released by the intense interaction of the surface with the plasma of the next pulse. Therefore, both metal and non-metal neutrals appear when intense metal plasma is produced.

One may speculate that the role of non-metal neutrals is reduced if the discharge was done in ultrahigh vacuum, and therefore somewhat higher charge states could be obtained. Though, non-metal neutrals can appear even in ultrahigh vacuum, for example, 
the hydrogen stored in the cathode itself (some metals can uptake rather large amounts of hydrogen). The production of metal neutrals in ultrahigh vacuum should be expected to be at least as significant as in high vacuum because the energy provided to plasmasurface interaction can now immediately act on the clean metal surface, producing neutral vapor. Therefore, the basic results of this investigation are not expected to change if ultrahigh vacuum was available.

In conclusion, it appears that the two counteracting processes, increasing ionization with increasing power on the one hand, and increased production of neutrals at high power on the other hand, result in a maximum of the mean ion charge state of about $6+$ to $7+$, which typically occurs at peak power of about 2-3 MW.

\section{ACKNOWLEDGMENTS}

This work was supported by the U.S. Department of Energy, Initiatives for Proliferation Prevention, under Contract No. DE-AC02-05CH11231 with the Lawrence Berkeley National Laboratory. 


\section{References}

1

2

3

4

5

(1)

(1)
A. A. Plyutto, V. N. Ryzhkov, and A. T. Kapin, Sov. Phys. JETP 20, 328 (1965).

W. D. Davis and H. C. Miller, J. Appl. Phys. 40, 2212 (1969).

I. G. Brown, Rev. Sci. Instrum. 65, 3061 (1994).

A. Anders, Phys. Rev. E 55, 969 (1997).

A. Anders, Cathodic Arcs: From Fractal Spots to Energetic Condensation (Springer, New York, 2008).

E. M. Oks, A. Anders, I. G. Brown, M. R. Dickinson, and R. A. MacGill, IEEE Trans. Plasma Sci. 24, 1174 (1996).

$7 \quad$ A. Anders and G. Y. Yushkov, J. Appl. Phys. 91, 4824 (2002).

8 M. M. M. Bilek, P. J. Martin, and D. R. McKenzie, J. Appl. Phys. 83, 2965 (1998).

9 J. Rosén, A. Anders, S. Mráz, and J. M. Schneider, J. Appl. Phys. 97, 103306 (2005).

10 A. Anders, E. M. Oks, and G. Y. Yushkov, J. Appl. Phys. 102, 043303 (2007).

11 G. Y. Yushkov and A. Anders, Appl. Phys. Lett. 92, 041502 (2008).

12 R. E. Honig and D. A. Kramer, RCA Rev. 30, 285 (1969).

13 H. S. Carslaw and J. C. Jaeger, Conduction of Heat in Solids, 2nd ed. (University Press, Oxford, 1959). 


\section{Tables}

Table I: Typical electrical parameters of the experiment (measured for a gold cathode, data for other cathode materials may vary): labels are self-explanatory; FWHM refers to full width at half maximum.

\begin{tabular}{|c|c|c|c|c|c|c|}
\hline \multirow{2}{*}{$\begin{array}{c}\text { Capacity } \\
(\mu \mathrm{F})\end{array}$} & $\begin{array}{c}\text { Max. } \\
\text { Voltage, } \\
(\mathrm{kV})\end{array}$ & \multicolumn{3}{|c|}{ Current } & \multicolumn{3}{|c|}{ Power } \\
\cline { 5 - 7 } & $(\mathrm{kA})$ & $(\mu \mathrm{s})$ & $(\mathrm{A} / \mathrm{s})$ & $(\mathrm{MW})$ & $(\mu \mathrm{s})$ \\
\hline 0.15 & 15 & 2.4 & 1.4 & $5.6 \cdot 10^{9}$ & 12 & 1.1 \\
0.45 & 8.4 & 4 & 1.5 & $8 \cdot 10^{9}$ & 15 & 1.2 \\
11.6 & 5.8 & 14 & 7 & $2.7 \cdot 10^{9}$ & 16 & 4.5 \\
\hline
\end{tabular}




\section{Figure Captions}

Fig. 1 Experimental setup for measuring and maximizing ion charge state in a short pulse discharge in vacuum.

Fig. 2. Discharge current, voltage, and power for gold discharges in vacuum for the three circuit situations; note the differences in scales. The curves are single-pulse examples that exhibit typical fluctuations caused by the non-stationary cathode processes in interaction with the supply circuit.

Fig. 3 Example of single-pulse discharge current (gold cathode) and ion beam current at the beam monitor $0.9 \mathrm{~m}$ downstream from the extraction system. The data were smoothed using the spline smoothing function.

Fig. 4 Typical TOF spectrum of the gold ion charge state distribution: $1.5 \mathrm{kA}$ peak current, with 2 pulses per second, storage capacitor $0.45 \mu \mathrm{F}$ with a charging voltage of $4 \mathrm{kV}$.

Fig. 5 Current dependence of the TOF spectrum for gold plasma using the circuit with the $0.45 \mu \mathrm{F}$ capacitor giving a FWHM of $1.5 \mu$ s of the discharge pulse.

Fig. 6 Mean ion charge state number (gold) as a function of discharge current: here we show the new data (1.4 $\mu$ s and $1.5 \mu$ s pulses) and compare with results obtained with the longer, $7 \mu$ s pulses.

Fig. 7 Mean ion charge state number (gold) as a function of the rate of current rise.

Fig. 8 Mean ion charge state number (gold) as function of peak power dissipated in the discharge gap. 


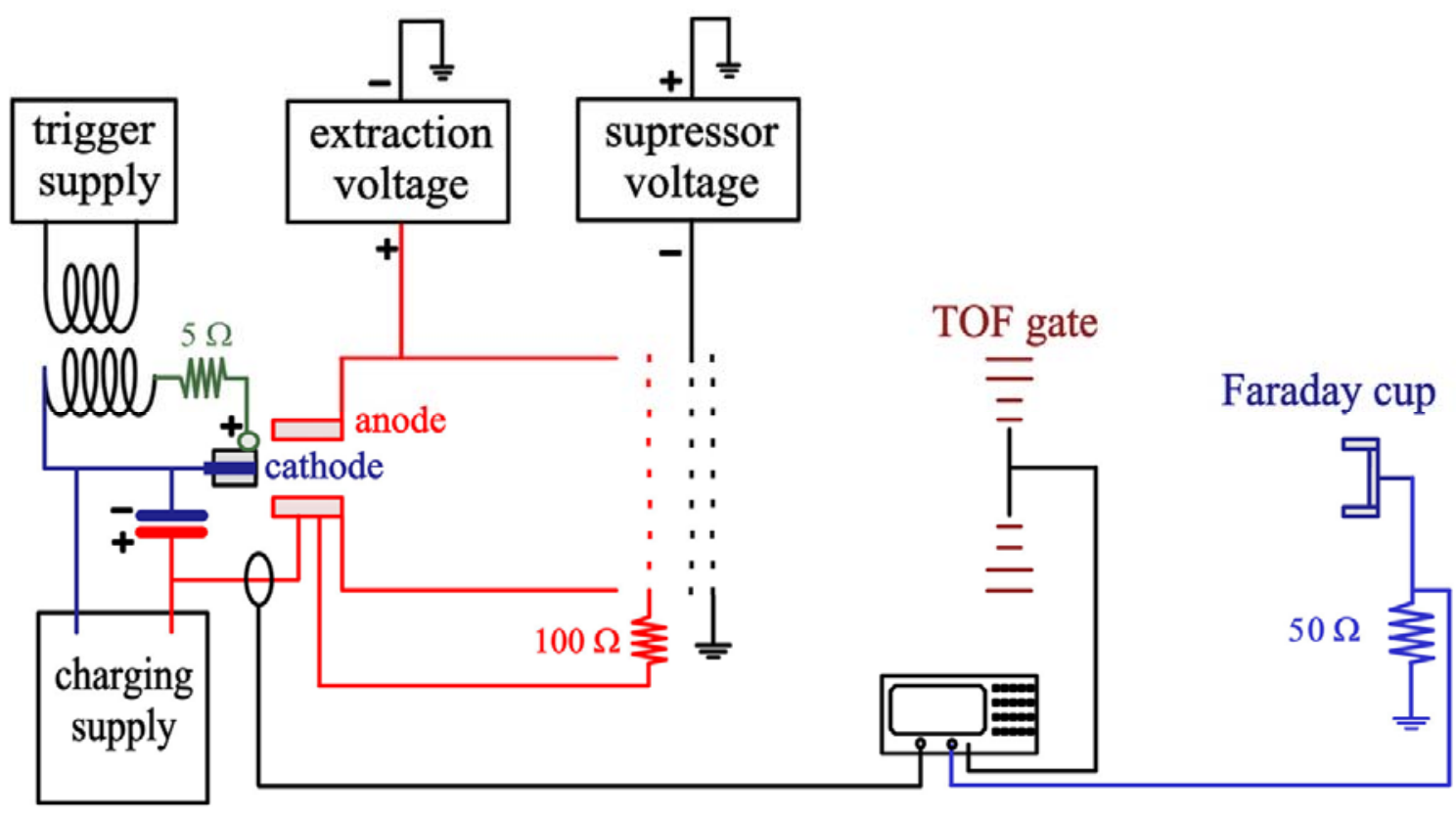

Fig. 1 

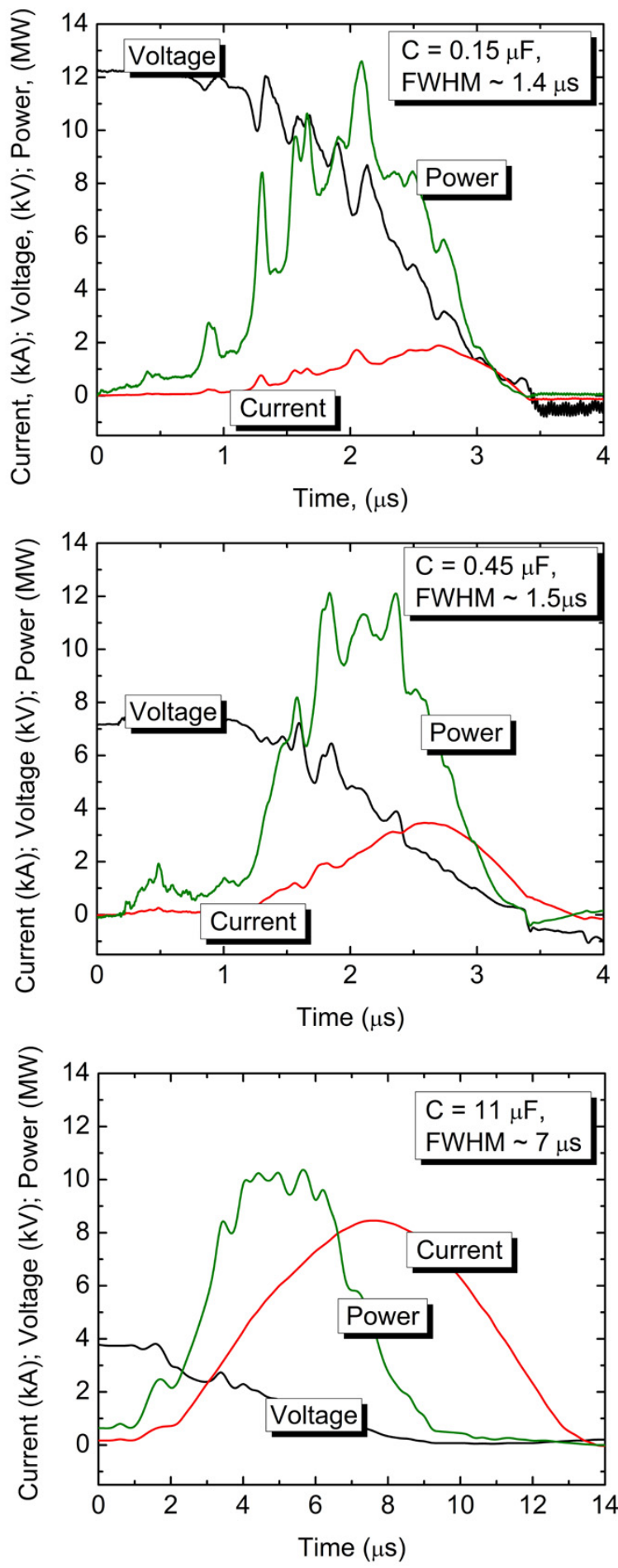

Fig. 2 


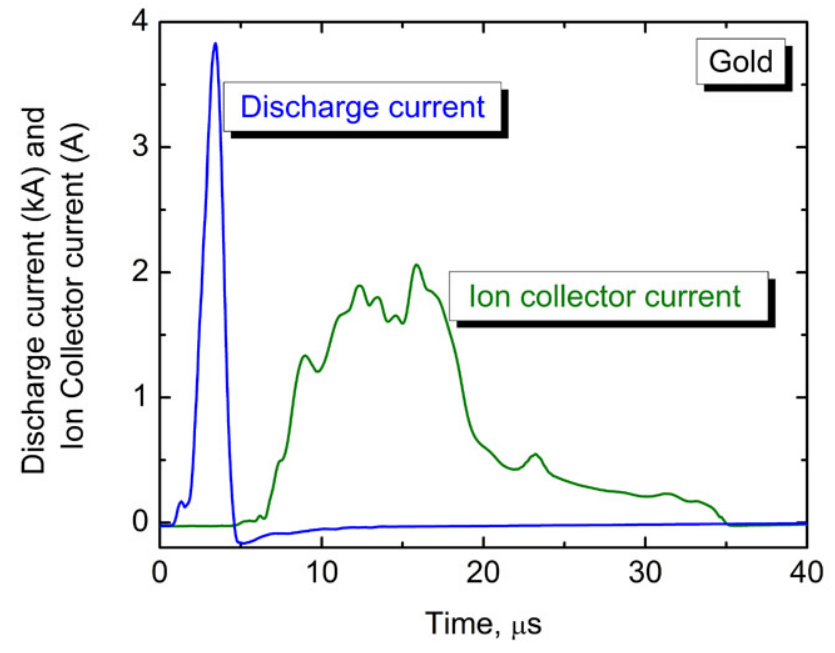

Fig. 3 


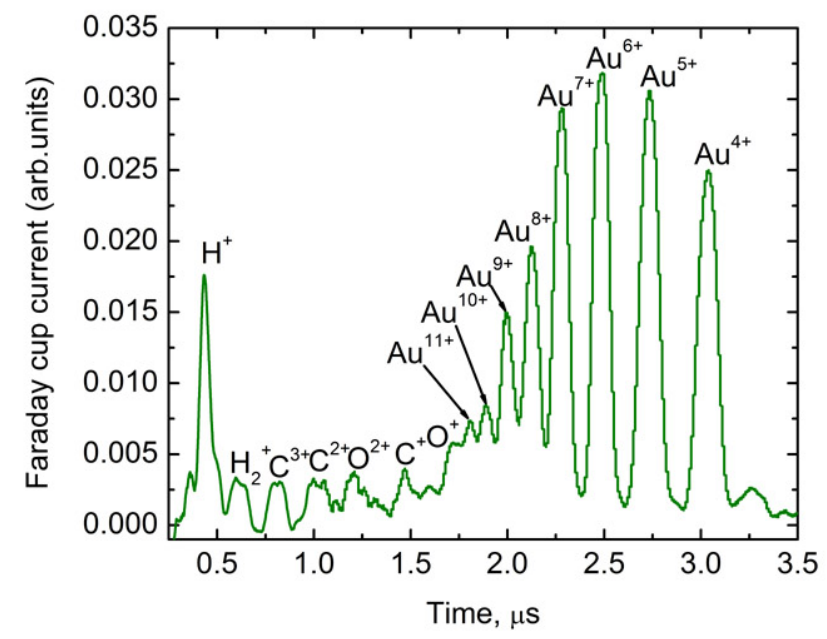

Fig. 4 


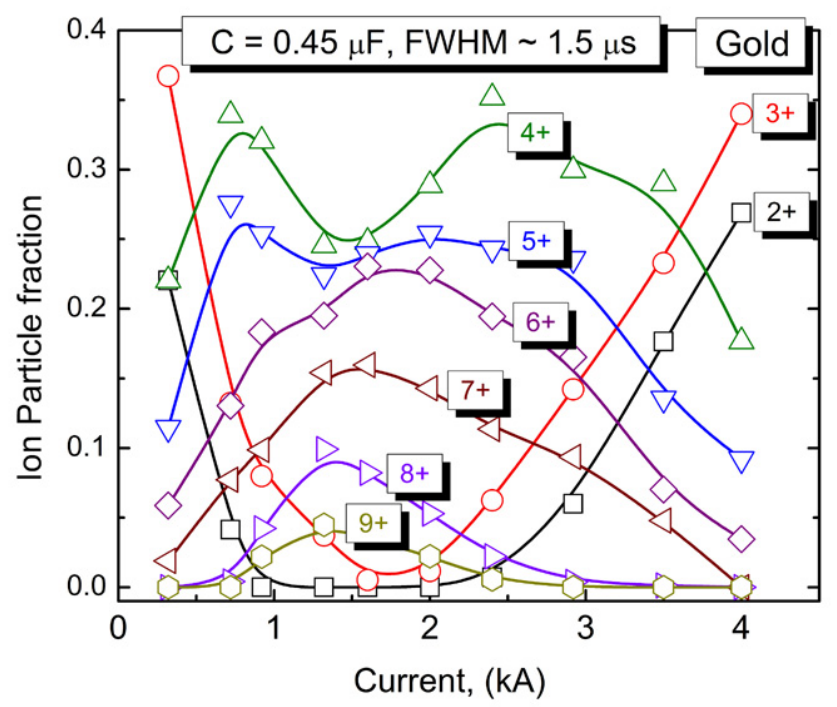

Fig. 5 


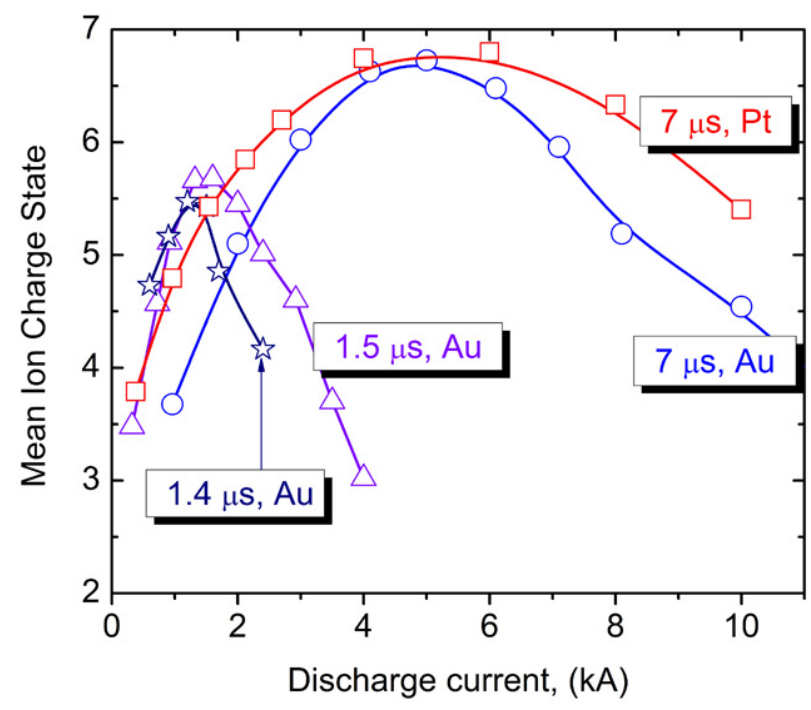

Fig. 6 


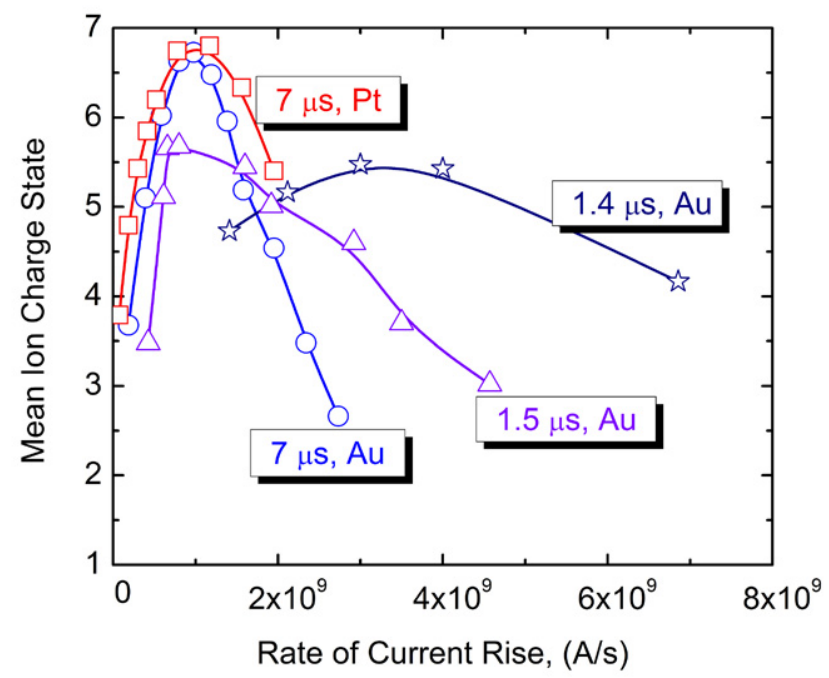

Fig. 7 


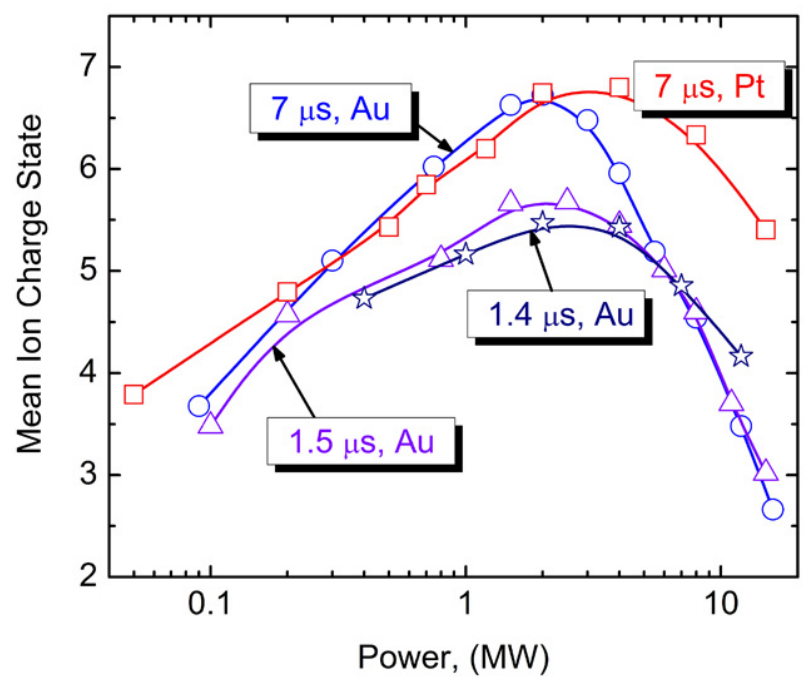

Fig. 8 